Historic, Archive Document

Do not assume content reflects current scientific knowledge, policies, or practices. 


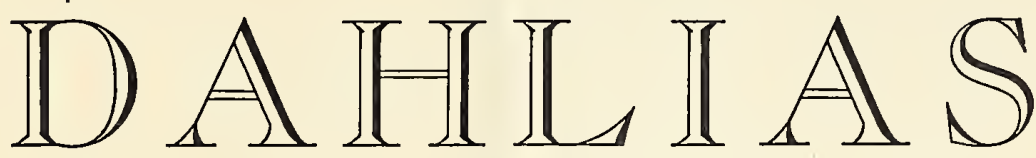

WHOLESALE LIST

To the Trade Only

SPECIAL OFFER FOR EARLY ORDERS

If payment in full is made at time of placing Early Orders (before February 1st) a cash discount of 10 per cent. or 15 per cent.

(Value in Stock) will be allowed

Note-After February 1st a stock value of 10 per cent. will be allowed for Cash Orders

Customer's selection for value of discount: 6 at 100 rate, less than 6 add 25 per cent to 100 rate prices.

Laocust Valley Dahlia $\mathbb{F}$ arm WM. A. FINGER, JR., Manager LOCUST VALLEY [ LONG ISLAND ] NEW YORK 


\section{TERMS TO THE TRADE}

Stock listed is strictly of our own growing for the trade and is not to be compared to surplus stock.

Prices quoted are (per 100) selected divisions showing eyes or sprouts, 6 at 100 rate, less than 6 add $25 \%$ to 100 rate prices.

We ship out only selected A. No. 1 divisions, which can be resold with the confidence that they will give satisfaction to your customers.

We don't substitute, and if sold out on a variety ordered cheerfully refund your remittance.

We harvested an exceptionally fine crop of bulbs, plump and firm, without signs of stunt or any other disease.

Transportation-All orders forwarded at purchaser's expense and risk and although we take special care in packing against freezing cannot guarantee against damage in transit.

Claims-No claims for rejections or deficiencies will be entertained unless made within ten days after arrival of goods.

GUARANTY. While we exercise the greatest care to have our stock true to label, and hold ourselves prepared to replace, on proper proof, all that may prove untrue, we do not give any warranty expressed or implied and in case of any error on our part, it is mutually agreed between the purchaser and ourselves that we shall not at any time be held responsible for a greater amount than the original price of the stock.

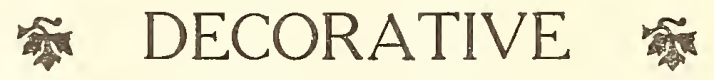

Per 100

AIDA-Deep maroon .............\$ 10.00

AMITYVILLE_Silver pink .......... 20.00

AMUN RA-Bronze, gold \& amber ...... 50.00

AUTUMN GLORY - Golden terra cotta .... 10.00

AZALEA-Creamy yellow \& pink ........ 15.00

BASHFUL GIANT-A pricot \& gold ....... 75.00

BEATRICE SLOCOMBE-Red, gold \& rose 25.00

BESSIE BOSTON-Deep scarlet ........ 12.00

CARMENCITA-Yellow striped red ...... 50.00

CAMBRIA-Light rose pink .......... 50.00

CHALLENGE-Yellow marked crimson .... 15.00

CLAUDIA TICE-Salmon, rose \& apricot .. 50.00

DAKOTA-Shade of flames .......... 15.00

DARLENE_Shell pink \& white ......... 10.00

DR. TEVIS - Salmon rose, gold \& apricot .. 20.00

DELICE_Rose pink ............... 15.00

DORO'THY ROBBINS-Best large flowering a utumn shade dahlia for high class florists' trade in cultivation. Color slightly darker than King of the Autumn. A seedling of same that always has perfect double blooms. Known in N. Y. City by Fifth Avenue Florists as Finger's Double King of the Autumn. No dahlia collections are complete without this sterling variety

DUSKY PRINCESS-Dark velvet .........

EDITH WOOSTER-Rosy red \& yellow ....

EVERETT WRIGHT-Dark salmon color

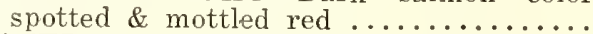

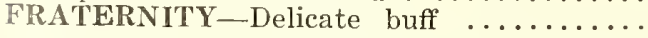

If you are not satisfied, don't hesitate to tell us. We want to make good. To succeed we must have your continued patronage. Our motto will always be SATISFACTION.

FLORENCE FINGER-Giant deep bloom of dark rose. In the N. Y. City flower market this dahlia is considered the BEST DARK PINK DAHLIA for reason of its strong straight stem, large size, good keeping qualities and wonderful color. Were we to grow only one dahlia, this

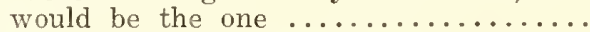

FOREST LOMA-Cerise pink \& canary yel-

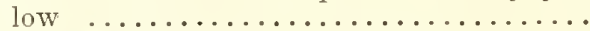

FRANK WALKER-Lavender pink ....... FUTURITY - Salmon rose \& pink ........ GLORY OF NEW HAVEN-Lavender pink

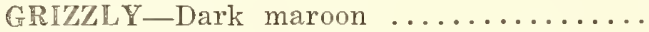
HATTIE FINGER-Deep dark rose pink ..

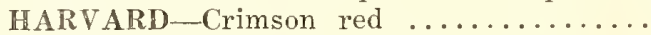
HOPE MODDLE-Dark buff \& yellow ..... HORTULANUS FIET-Salmon pink \& rose HOWARD WRIGHT-Light pink ........

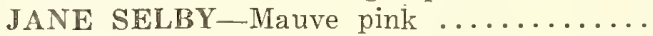
JENE CHARMENT-Lilac pink with lighter

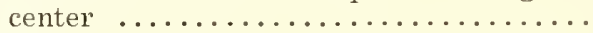

25.00

10.00

25.00

50.00

10.00

JERSEY'S BEAUTY - The finest true pink

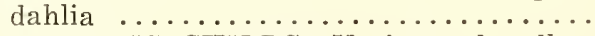

JOHN LEWIS CHILDS_Variegated yellow

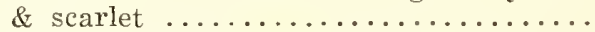
JUDGE MAREAN-Orange yellow \& gold .. KING OF COMMERCE-Tango \& gold ....

For a real better Autumn Shade Decorative Dahlia try Dorothy Robbins. A wonderful lasting cutflower, known by New York Fifth Avenue Florists as FINGER'S DOUBLE KING of the Autumn.
20.00

10.00

5.00

50.00

20.00

50.00

25.00

20.00

15.00

40.00

15.00

25.00

15.00

75.00

25.00

50.00

35.00 


\section{DECORATIVE (Continued)}

Per 100

LE GRAND MANITOU-White \& purple variegated $\ldots \ldots \ldots \ldots \ldots \ldots \ldots \ldots$.

MARCELLA-Seashell pink $\ldots \ldots \ldots \ldots \ldots$

MILLIONAIRE-Lavender pink ...........

MINA BURGLE-Rich dark scarlet .........

MINUTE MAN - Variegated deep orange, shading to yellow center \& heavily tipped

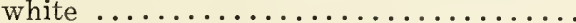

MME. LUMIER - White tipped reddish violet MONS. LE NORMAND-Y ellow tipped white MRS. ATHERTON-Light yellow .......... MRS. CARL SALBACH-Deep lavender pink MRS. E. R. HEIM-Salmon pink, rose \&

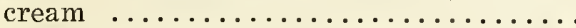

MRS. F. W. SCHEIFF-Golden bronze \& orange $\ldots \ldots \ldots \ldots \ldots \ldots \ldots \ldots \ldots$

MRS. I. DE VERE WARNER-Rich mauve pink

MRS. NAT. SLOCOHBE-Bright golden yel-

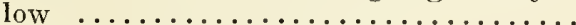

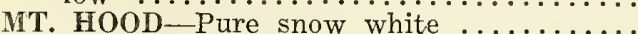
PATRICK O'MARA - Orange buff \& rose .

PAUL MICHAEL (Calif. Gold)-Orange buff reverse old rose ..................

PERFECT BEAUTY - Scarlet \& white .... PRIDE OF CALIFORNIA-Rich crimson . . PRINCESS PAT - Old rose color .......... PURPLE MANITOU-Deep violet purple...
10.00

10.00

25.00

6.00

15.00

10.00

35.00

15.00

25.00

25.00

20.00

40.00

20.00

10.00

30.00

50.00

35.00

15.00

40.00

5.00
Per 100

10.00

40.00

40.00

15.00

ROSEM A IVR - Rich rose pink .....................

SAMPSON-Variegated bronze scarlet \&

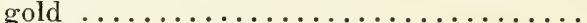

SAMMIE-Deep primrose yellow .........

SAN MATEO-Variegated yellow \& scarlet

SCOTTISH CHIEF-Golden amber ........

SEBASTAPOOL-Deep orange ...........

SEABRIGHT-Bright orange...$\ldots \ldots \ldots$

SNOWDRIFT_Giant pure white .........

SOUV. DOAZON-Brick red ...............

STUNNER_Giant light yellow ............

SULPHERIA-Clear sulphur yellow .......

SUNSHINE-Scarlet \& gold .............

SWIFT-Immense sulphur yellow ..........

SYLVIA DICKEY - Large free blooming of phlox pink color. Most profitable light pink dahlia for florists' use. A gem for garden and large enough for exhibition TERRA COTTA-Terra cotta color ....... THEO. VAIL_Old gold \& apricot ......... THE EMPEROR-Giant deep maroon ...... VENUS - White \& lavender ............. WHITE SWAN-Florists' favorite white .. YELLOW COLLOSSE-Bright yellow .....
40.00

10.00

40.00

15.00

10.00

10.00

35.00

15.00

25.00

25.00

25.00

20.00

20.00

15.00

15.00

25.00

25.00

5.00

15.00

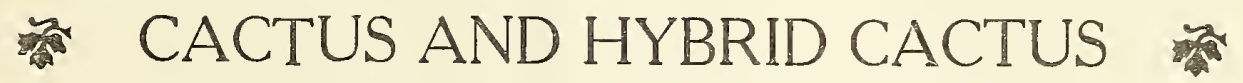

ATTRACTION-Delicate lavender pink ...\$25.00

BIANCA_Rose lavender pink .......... BERTHA HORNE-Bronzy yellow \& apricot COLOSSAL PEACE-Creamy white \& pink

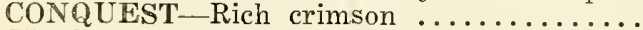

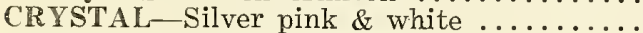
DIANA - Crimson \& violet .............

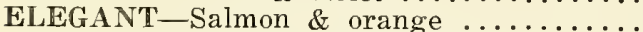
ETENARD DE LYON_Carmine rose ..... F. W. FELLOWS-Bright orange ......... GARIBALDI-Brilliant scarlet shading dark-

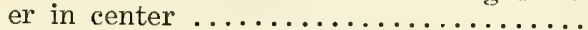

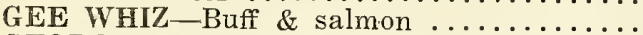
GEORGE IVALTERS-Salmon pink \& gold GLADYS SHERWOOD-Pure white ...... GOLDEN GATE-Golden yellow \& bronze ...

JEAN CHAZOT (Gay Paree)-Golden bronze

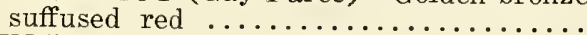

JOHN RIDING - Rich crimson $\ldots \ldots \ldots \ldots \ldots$

KALIF_-Pure scarlet ...............

LADY HELEN_Carmine rose \& cream ....

MARATHON-Deep rich purple ..........

MARGARET BOUCHON-Pink \& white center

15.00

15.00

15.00

15.00

15.00

10.00

20.00

12.00

20.00

50.00

20.00

30.00

50.00

15.00

35.00

15.00

25.00

75.00

10.00

15.00
MELODY_Bright yellow \& white ....... 1000 MRS. EDNA SPENCER-Lavender pink ... 25.00 MRS. W. E. ESTES-Pure white ....... 35.00 MT. SHASTA-Light shell pink \& yellow .. 15.00 MRS. WARNAAR - Creamy white \& pink .. 35.00 NIBLUNGHORT-Old rose \& apricot ..... 35.00 PIERROT-Amber tipped white ........ 20.00 QUAKER CITY-Primrose yellow ........ 10.00 RED CROSS_Scarlet \& gold ......... $\mathbf{5 0 . 0 0}$ RHEINKONIG-Pure white ........... 12.00 RHEINSHER FROSHIN-White \& carmine

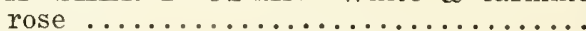
SPRINGFIELD-Amaranthine red ......... THE LION_-Bronzy rose \& salmon ........ 10.00 U. S. A.- Orange buff .............. 75.00 WAR DANCE-Scarlet \& gold ........... 10.00 WOLFGANG VON GOETHE-Apricot \&

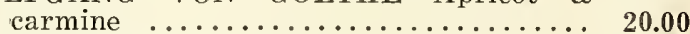
WODAN-Salmon rose ........... 25.00 YELLOW PRINCE-Bright yellow ........ 15.00

Our Dahlias are unpropagated and are grown from roots, not cuttings, so are free from weakness or disease. 


\section{PEONY AND PEONY DECORATIVE}

Per 100

ADMIRAL DEWEY-Bronzy yellow \& red . . AURORE-Deep rich bronzy orange ..... BILLIONAIRE-Golden orange .......... GEISHA-Scarlet \& gold .............. GENERAL CUSTER-Yellow, white \& coral

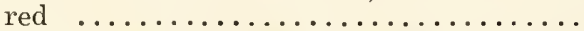

HOLMAN HUNT-Deep crimson shaded

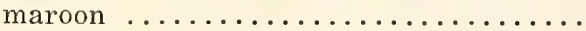

KING OF THE AUTUMN-Autumn shades
15.00

15.00

25.00

50.00

10.00

15.00

35.00
LORD MILNER-Creamy yellow gold \&

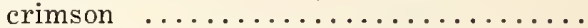

MEYERBEER-Fascinating purple .......

MRS. JESSIE SEAL-OOld rose \& golden

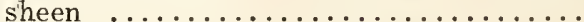

MRS. JOHN BRAY-Variegated white \&

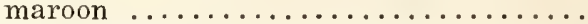

OREGON BEAUTY-Bright scarlet ......

PRIDE OF PORTLAND-Brighter Geisha

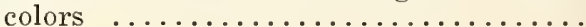

WASCO BEAUTY-Pretty Geisha colors ...
Per 100

15.00

25.00 .

20.00

25.00

10.00

25.00

15.00

\section{SHOW OR BALL TYPE}

A. D. LIVONIA-Delicate shell pink ...... \$ AMERICAN BEAUTY-Deep wine crimson AMERICAN FLAG-Variegated red \& white ELM CITY - Bronzy yellow, amber \& red .. ELSIE BURGESS-White lavender \& rose

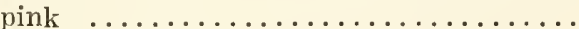
GOLDEN OPPORTUNITY-Buttercup yel-

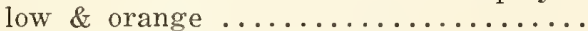
HELEN HOLLIS-Scarlet red ............ J. K. ALEVANDER — Violet purple ....... LONG ISLAND SUNRISE-Scarlet \& gold .
5.00

10.00

10.00

15.00

8.00

15.00

12.00

25.00

50.00

MARY BOWMAN-Golden yellow tinged

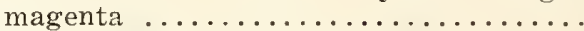
MADE ADAMS-White \& clear pink MRS. J. P. SMITH-Rich cherry red .... 10.00 NORMA - Bright orange buff ......... 15.00 POLLY UPHAM_Beautiful pink shades ... 10.00

TESTIMONIALS

A letter to our manager from MR. DARNELL, the donor of the famous prize, THE DARNELL CUP, that he is annually offering at New York Show for best commercial dahlias as an inducement for hybridizers to produce new dahlias having better commercial merits. It's a safe bet that any dahlia recommended by Mr. Darnell is a good one for he knows and appreciates good reliable dahlias.

\section{THE DAHLIA FARM \\ (On the Sunrise Trail)}

East Moriches, Long Island, N. Y. January 31st, 1925

Locust Valley Dahlia Farm,

Locust Valley, L. I., N. Y.

Dear Mr. Finger:

If you look at my 1924 catalogue you will see what I said of your seedlings, and you can say that "Sylvia Dickey" is a wonderful cut flower variety, far superior to Venus. Anyone that can introduce a dahlia as good as the "Florence Finger" has benefited the flower world to a far greater degree than any of the hybridizers who work to produce a rival of the cabbage for size.

Those three dahlias, Dorothy Robbins, Florence Finger and Sylvia Dickey, will keep your memory green and will be listed by every wholesaler as soon as they are cheap enough to be within reach of the purely commercial grower. With all best wishes I am

Sincerely yours,

(Signed)

DARNELL.

\section{MRS. HOWARD HOLSINGER \\ Dahlia Specialist, Denton, Md.}

May $29 / 26$

Your letter received also the dahlia tubers, and I want to say your tubers are mostly better than those I have gotten elsewhere, in fact I'll take my hat off to you; the greater part of my dahlias come from you.

\section{N. F. FLITTON SONS, Baltimore, Md.}

May 14 th $/ 26$ Dahlia roots received in fine shape and entirely satisfactory. Order O. K., thanking you for liberal count.

HACKS NURSERIES

Winnipeg, Man., Canada.

Aug. $23 \mathrm{rd} / 26$

We were well pleased with your stock. (it being best we have seen) and we would be very pleased to have an advanced list of the varieties you will have for sale at the earliest date.

\section{THE HAVICE NURSERIES,}

West Park, Cleveland, $\mathrm{O}$.

Jan. $28 / 26$

We got a number of bulbs from you last year and were very much pleased with the quality of them and the way they were packed. They all grew and we got good results with the blooms.

(Signed) LEONE HAVICE.

